\title{
What Palliative Care can learn from Geriatric Medicine?
}

Hannah Enguell, Academic Clinical Fellow ${ }^{1}$

Rowan H Harwood, Professor of Palliative and End-of-Life Care and honorary consultant geriatrician ${ }^{1,2}$

1. Nottingham University Hospitals NHS Trust

2. School of Health Sciences, University of Nottingham, NG7 2UH

Corresponding author Prof Harwood; Rowan.harwood@nottingham.ac.uk; 01158230873

\begin{abstract}
Most people die when they are old, with multiple pathologies, and while living with frailty or dementia. These circumstances need the specialist skills of geriatric medicine. Death may not be unexpected, but survival and restoration of function are usually uncertain, influencing the approach to medical intervention. Assessment considers medical, functional, mental, social, and environmental domains. Care requires a mix of acute, rehabilitation, mental health and palliative expertise, and evolves with changing circumstances. Relief of suffering and maintenance of function are key goals, but not the only ones. Mental distress is as common as physical; investigation- and treatment-burden are important; drug treatments are prone to adverse effects. A focus on person-centeredness rather than the end-of-life is needed. This prioritises respect for individual diversity in needs, assets and priorities, and rigorous decision-making, to achieve what is the right intervention for that person at that time.
\end{abstract}




\section{Key Points}

- People living with frailty, multi-morbidity, dementia and chronic organ failure are prone to deterioration and crisis, following which outcome is uncertain: they may or may not survive

- Comprehensive Geriatric Assessment considers medical, functional, mental, social and environmental domains, which helps manage the complexity

- Person-centred care emphasises respect for individual diversity, in particular in preferences and priorities

- We should aim to choose the right intervention for each individual, at the time of making the decision, living with uncertainty, and changing plans as events evolve

- Palliative and geriatric medicine can valuably learn from each other.

\section{Short introduction}

When frail older people fall ill, we need clarity in what we are trying to achieve. Patients' and their families' opinions vary greatly, so the right approach will often be different from one patient to the next. Geriatric medicine works to integrate acute, rehabilitation, mental health and palliative perspectives.

\section{Introduction}

The specialty of palliative care developed to meet the needs of people dying from cancer, in particular, the need to relieve severe pain. From this developed a sophisticated approach to control of a range of distressing symptoms, based on meticulous assessment, and insightful clinical pharmacology. It recognised the need for open and honest communication, and attention to psychological, social and spiritual concerns.

The original remit of palliative care was for people approaching the last few days or weeks of their lives, in circumstances where the prognosis and trajectory were fairly clear. Planning for the future included discussions about preferred place of death (in particular, enabling death at home), reducing treatment burden and prioritising treatments aimed at relief of symptoms over those intended to prolong life. Palliative care has been singularly successful in working across healthcare boundaries, including specialities treating cancer, acute medicine, and supporting people living in care homes and their own homes.

Tensions have emerged, however. Cancer survivorship has increased, with the development of new and aggressive therapies. It may be unclear whether a cancer is survivable, leading to 'twin-track care', in which palliative approaches are undertaken alongside potentially lifeprolonging therapies. Several trials have suggested that in advanced cancer, survival with palliative care may exceed that with active treatment [Temel et al, 2010; Reljic et al, 2017]. Consequently palliative care is increasingly seen as a 'supportive' adjunct to other therapies.

Cancer incidence increases with age, and most people dying with cancer are old. Older people are rarely affected by a single pathology. Often the effect of multiple co-morbidities may equal or exceed that of an accompanying cancer (so 'performance status' may not reflect cancer prognosis). Judging prognosis in people suffering organ failure or frailty is 
notoriously difficult, making it uncertain when to adopt a palliative approach [Elliot et al 2017].

Changing epidemiology drives the scale of these dilemmas. There are 11.8 million people over the age of 65 living in the UK, and 1.6 million over the age of 80 . Of the 525,000 deaths in $2017,85 \%$ were in those aged over 65 . Only $28 \%$ of people die from cancer. The commonest certified cause of death for women in the UK is now dementia; one in three people have dementia when they die. Age-specific rates of death from ischaemic heart disease and stroke have reduced dramatically, through changes in incidence, reduced casefatality and better supportive care. Death from chronic lung, heart, renal and neurological conditions display different trajectories to that in cancer, with prolonged periods of poor health over many months or years, punctuated by acute crises which may recover to some extent.

Supporting people living with frailty or dementia are among the biggest challenges in contemporary healthcare. Most people with these conditions die in hospital or nursing homes. Mortality for an older person admitted to hospital as an emergency is $25 \%$ at 90 days, $30 \%$ at six months, and $40 \%$ at a year [Bradshaw et al 2013; Reynish et al, 2017]. Median survival for someone admitted to a nursing home is less than a year, and many die within the first few months (having moved there due to failing health). Yet median survival in the population at age 90 is five years [Office for National Statistics, 2018]. Medical care is usually provided by general practitioners, acute and general hospital physicians, and geriatricians. The numbers requiring end-of-life care in old age indicate that a joint approach is required; only $15 \%$ access specialist palliative care [Gott et al, 2013]. All geriatricians look after people who are dying, and many are comfortable with the principles and practice of palliative medicine, although levels of expertise and interest vary.

\section{How geriatric medicine works}

The term 'geriatric' is disliked by older people, so the specialty exists under numerous synonyms, such as medicine for the elderly or healthcare for older people. The Royal College of Physicians (London) describes Geriatric Medicine as: 'a branch of general medicine that is concerned with the clinical, preventative, remedial and social aspects of illness in old age. The challenges of frailty, complex co-morbidity, different patterns of disease presentation, slower response to treatment and requirements for social support call for special medical skills.' Geriatric medicine is broader than its parent discipline, requiring expertise in, for example, acute medicine, psychiatry, neurology, rheumatology, urology, therapeutics, rehabilitation, ethics and law. Delivery invariable involves a multidisciplinary team, including physiotherapy, occupational therapy, speech and language therapy, nursing and social care professionals.

\section{Comprehensive geriatric assessment (CGA)}

Geriatric medicine is systematised within a framework, known as Comprehensive Geriatric Assessment (CGA), comprising five dimensions (table 1). The output is a multi-professional management plan. Such plans are rarely, knowingly, labelled 'palliative care', but they are 
often just that, to all intents and purposes. Geriatric medical wards provide the workforce needed to deliver CGA.

Trial evidence shows that this approach reduces mortality, disability, and need for care home placement and increases chances of returning and remaining at home [Ellis et al, 2017]. A range of goals are addressed (table 2), and models of care deployed (table 3 ). For example, person-centred care requires attention to the experience of care as well as the outcome, reflected in communication style, decision-making, therapeutic compromises and de-prescribing, engagement of families and other carers, and the discharge planning. This is important especially in dementia and at the end of life.

\section{Rehabilitation}

Restoration of function in older people, following acute illness, injury, or associated with chronic disability, requires explicit effort. This can mean intervening at the level of the disease (potentially curative), body systems (relieving symptoms, increasing muscle strength and cardiorespiratory fitness), abilities (through functional training, aids or appliances) and adapting the environment. Rehabilitation can be started very early in an illness, in an attempt to prevent deconditioning, complications and further loss of function, and may be required after even a minor illness. The process may take many months; in the face of severe illnesses with uncertain recovery, including delirium, stroke or hip fracture, or with co-morbid dementia, this requires attention to communication, psychology, the negotiation of achievable goals and the avoidance of both dis-spiriting futile attempts, but also therapeutic nihilism ('no rehabilitation potential').

Inter-specialty liaison

Geriatricians often help manage patients with hip or other fractures (ortho-geriatrics), major trauma, elective surgery or with cancer. This was predicated on the association between comorbidities and risk of mortality, and the need for explicit rehabilitation, and has been demonstrated to improve outcomes [Roche et al, 2005; Partridge et al, 2017]. Intervention may be anticipatory, but will also focus on the illest patients peri-operatively, including those who may die. There is a close working relationship with old age psychiatry.

\section{Community geriatrics}

Attention given to older people living at home and in care homes has varied over time, with changing demands from acute medical duties, development of different commissioning arrangements, and a concern to maintain access to acute hospital facilities for frail older people when needed. In recent years, community geriatrics has seen a resurgence, working alongside community matrons, care homes and intermediate care. This has included promoting advance care planning, and negotiating appropriate medical care for frail older people approaching the end-of-life.

Different geographical localities have evolved different systems. Fragmented services, poor continuity and lack of shared records have been major challenges to care delivery, especially when trying to support preferences to avoid hospital admission. Planning conversations may 
be initiated at opportune moments, never to be fully followed-though, or communicated to other stakeholders.

\section{The health of older people towards the end-of-life}

\section{Frailty}

Geriatricians primarily look after people who are frail. Frailty is a 'propensity to decline in the face of a stressor' [Clegg et al, 2013]. It is a health state, related to the ageing process, in which body systems lose their built-in reserves, and ability to maintain homeostasis. Around $10 \%$ of people aged over 65 years are frail, rising to between a quarter and a half of those over 85 years. People living with frailty are vulnerable to crises, loss of well-being and function, falls, hospital and care home admission, and death, in the face of acute illness, injury, treatment complications, adverse effects of drugs, disruption of social circumstances or change in environment. Frailty may be defined by presentation (delirium, falls, acute immobility), co-morbidities (dementia), place of residence (care homes), or an assessment score or scale (such as Rockwood's Clinical Frailty Scale, Edmonton [Rolfson et al, 2006] or electronic Frailty Index [Clegg et al 2016]).

\section{Trajectories}

Murray et al (2005) described three trajectories of decline at the end-of-life; the rapid decline associated with death from cancer, steady decline punctuated by exacerbations and recovery in organ failure, and a slow inexorable 'dwindling' in frailty and neurodegeneration. A fourth trajectory typical for stroke (or hip fracture) has been proposed, with sudden decline followed by increased vulnerability amongst survivors [Creutzfeld et al, 2015]. Others have described different trajectories with wild and dramatic swings between near-death and relative recovery, for example in heart or respiratory failure [Goodlin 2009]. A similar pattern is often seen in frailty and dementia. Acute illness or injury can lead to a dramatic, but potentially recoverable, decline in function, especially when complicated by delirium [Jackson et al 2017].

'Transition points' can be identified within these trajectories, when a discussion about future care may be welcomed or necessary, including a change from active management to palliative care. Examples are retirement, hospital admission, diagnosis of dementia, an episode of delirium, and care home entry. Recognising these transition points and clarifying the issues (diagnosis, prognosis, management options, preferences), amidst the complexity of nonspecific presentations, multi-morbidity and polypharmacy, functional and mental decline, and social vulnerability, is central to geriatric medical practice.

\section{Acute medical care of frail people}

People with frailty are prone to crises, which lead to frequent use of emergency departments and acute medical admissions. Two-thirds of emergency admissions are of people over the age of 70 [Goldberg et al 2012]. Geriatricians often take part in all-age admission rotas, but increasingly 'acute frailty services' attempt to apply the principles of CGA at the point of hospital admission. Geriatrician-led decision-making allows more informed assessment and consultation with stakeholders, decisions about appropriate and 
inappropriate treatment, early access to community alternatives and place of death. In the face of a crisis, acute severe illness and physiological derangement, and in the absence of information to guide otherwise, a presumption of intervention and attempt at 'rescue' is often appropriate (for example, aggressive hydration and intravenous antibiotics). But this should not become a default, and efforts should be made to assess when treatment is, or is not, likely to deliver benefit, and ascertain what is, or is not, wanted care.

Hospital admissions are at best disruptive for frail older people and people living with dementia, often being frightening and disorientating, leading to distress, delirium, deconditioning and loss of abilities [Porock et al, 2014]. Avoiding hospital admission, whilst maintaining access to wanted investigation and hospital-based therapy is an important approach to preventing iatrogenic decline. That said, there is little prospect of a reduction in demand for acute hospital care for older people, including those with dementia, and a major effort is required to adapt the environment, staff skills, and care processes to make them appropriate for frail older people, including those who are dying.

\section{Dementia care}

Half of older people admitted to hospital have cognitive impairment, including $40 \%$ with dementia and a third with delirium (with considerable overlap between the two). The impact of disruption and decompensation is particularly severe, and levels of distress can be high. Up to half have pain, but other distressing symptoms such as fear, delusions and hallucinations are also important, and often not ascertained by hospital physicians unused to examining the mental state [Goldberg et al, 2012].

A biopsychosocial model of care, called person-centred dementia care, incorporates four dimensions [Brooker 2007]:

- Valuing the person and those who care for them

- Individualising care (including physical and mental health, abilities and disabilities, biography and personality, preferences, wishes and resources)

- Empathic understanding (seeing the person's point of view)

- Using relationships to provide comfort, attachment, inclusion, occupation and affirming identity.

Dementia-friendly or specialist wards are distinct from other acute medical wards in their layout, staffing and functionality [Goldberg et al, 2013]. They should be aesthetically calming with appropriate lighting, cues to time, date and place, purposeful activities and flexible schedules, including open visiting for relatives and carers. They emphasise knowing about biography, as meaningful interaction is easier when staff understand a person's past and personality. Anyone who interacts with older people will relate to the idea that at some stage in our lives we live more in the past than the present.

Explicit palliative care for people with dementia is under-developed nationally. Ideally, plans for the future (advance care planning) should be made early after a diagnosis, by those who wish to engage, whilst they retain sufficient cognitive ability and mental capacity, part of the rationale for early dementia diagnosis. Many will make a will, or appoint a lasting power of 
attorney, but relatively few want to make specific medical plans. People vary in their outlook on the future [Hill et al, 2017]. Some want as much autonomy and control as possible. Others look to family both to make decisions and provide care. Others do not want to contemplate an uncertain future, rather celebrating their ability to cope in the present in the face of adversities [Nicholson et al, 2012]. Others still are pragmatic, worrying more about whether needs are met, than about what care is provided or where. As the end-of-life approaches, care provision may be very similar to that required by any frail older person. Available support varies, but is generally under-provided and hard-pressed, often lacking in key areas such as care at night, respite care, carer and bereavement support.

\section{Delirium}

Delirium is an acute neuropsychiatric syndrome caused by physical illness, injury, drugs or drug withdrawal. It is common, generally under-recognised, distressing and dangerous. Prognosis is poor (40\% are dead by six months [Siddiqi et al, 2006; Whittamore et al, 2014]), and it may have a prolonged recovery time (30\% unresolved at one month, $25 \%$ at three months, $20 \%$ at six months [Coles et al, 2009]). Hypoactive delirium has an especially poor prognosis. Delirium may not resolve at all, being a mechanism for progression of dementia.

Predicting whether delirium will progress to death directly (e.g. individuals stop eating and drinking), indirectly (e.g. secondary to pneumonia), or due to the underlying medical illness, is almost impossible to determine. Anecdotally, families and carers report recurrent end-oflife discussions and unexpected recovery, but each episode becomes harder to recover from. Communicating and making treatment decisions in the face of this uncertainty, whilst delivering ethically-sound care, requires great skill.

\section{How geriatric and palliative medicine are alike}

Both geriatric and palliative medicine care work in the context of diseases which are chronic, progressive or incurable, with crises that are due to intercurrent illness or injury, complications or disease progression. Both require a problem-based approach, thorough and systematic assessment and diagnosis, multidisciplinary therapy, and working in the face of uncertainty. Emphasis on communication, family engagement, explicit decision-making and careful clinical pharmacology are shared. The person-centred dementia care philosophy, with its emphasis on individualisation, respect for preferences and wishes, concern for emotional care and use of relationships to support identify and provide comfort has particular resonance in palliative medicine. There is no fundamental conflict or competition between the two specialties.

\section{A vision for supportive and palliative care for frail older people}

The medical care of frail older people who may be dying is necessarily different from that required by people dying with cancer. Death is not unexpected, but in many cases outcome will be uncertain, both in terms of survival and restoration of function. The predominant source of distress is mental (delusions, hallucinations, anxiety, delirium, dementia), rather than pain, although this and other symptoms are common too. Psychotropic and analgesic medications are all prone to adverse effects on older and cognitively-impaired patients, and 
must be used with caution when death may yet be a way off. Suffering and loss of function are key problems, and their relief are key goals.

For many or most patients, at the point of assessment and care delivery, it will be uncertain if survival will follow or not. Palliative approaches should therefore be integrated with all care. Much, but not all, apparently 'aggressive' medical intervention (such as intravenous fluids and antibiotics, and some enteral tube feeding) reflects uncertainty, and treatment choices and preferences determined as part of a consultation or 'best interests' assessment, as required by mental capacity law.

Care is 'eclectic': a mix of different things: acute, rehabilitation, mental health and palliative. In many cases the objective of care is resolution of the acute injury or illness, which is expected, wanted and hoped for. In other cases, mental symptoms are predominant, requiring combinations of drug and non-drug approaches, and mental health service followup. Explicit rehabilitation is required where function has been lost, or disability is causing problems, although a balance must be drawn with futile attempts in those too frail or disabled to benefit. Engagement of families and carers, and advance care planning are important, although during or in the immediate aftermath of a crisis, or after a new diagnosis of dementia, may not be the best time to make plans.

If there is a single lesson from geriatric medicine, it might be to focus on personcenteredness, rather than palliation or the end-of-life per se. Person-centredness prioritises respect for individual diversity, and rigorous decision-making, on the basis of what is the right intervention for that person at that time, in the face of uncertainty. That may look like conventional palliative care, or it may look like acute medicine, but it will consider multiple domains (medical, functional, mental health, social, environmental), and be flexible enough to compromise in evolving circumstances (such as changing information, or poor response to treatment). Success is rarely judged by the 'saving of life', rather the relief of distress, maximising of function and quality of life, and respect for identity and preferences.

\section{REFERENCES}

Bradshaw L, Goldberg SE, Whittamore K, et al. Six month outcomes following an emergency hospital admission for older adults with co-morbid mental health problems indicate complexity of care needs. Age and Ageing 2013; 42:582-588

Brooker D. Person-Centred Dementia Care: Making Services Better. London: Jessica Kingsley Publishers; 2007.

Clegg A, Young J, Iliffe S, Rikkert MO, Rockwood K. Frailty in elderly people. Lancet 2013, 381: 752-762.

Clegg A, Bates C, Young J, et al. Development and validation of an electronic frailty index using routine primary care electronic health record data. Age and Ageing 2016; 45: 353-360.

Cole MG, Ciampi A, Belzile E, Zhong L. Persistent delirium in older hospital patients: a systematic review of frequency and prognosis. Age and Ageing 2009; 38: 19-26. 
Creutzfeld CJ, Longstreth WT, Holloway RG. Predicting decline and survival in severe acute brain injury: the fourth trajectory. BMJ 2015; 351:h3904

Elliott M, Nicholson C. A qualitative study exploring use of the surprise question in the care of older people: perceptions of general practitioners and challenges for practice. BMJ Supportive \& Palliative Care 2017; 7: 32-38.

Ellis G, Gardner M, Tsiachristas A et al. Comprehensive geriatric assessment for older adults admitted to hospital. Cochrane Database of Systematic Reviews 2017, Issue 9. Art. No.: CD006211. DOI: 10.1002/14651858.CD006211.pub3

Goodlin SJ. Palliative Care in Congestive Heart Failure. Journal of the American College of Cardiology 2009; 54: 386-396.

Goldberg SE, Whittamore K, Harwood RH et al. The prevalence of mental health problems amongst older adults admitted as an emergency to a general hospital. Age and Ageing 2012; 41: 80-86.

Goldberg SE, Bradshaw LE, Kearney FC, et al. Comparison of a specialist Medical and Mental Health Unit with standard care for older people with cognitive impairment admitted to a general hospital: a randomised controlled trial (NIHR TEAM trial). BMJ 2013;347:f4132

Gott M, Ingleton C, Gardiner C, et al. Transitions to palliative care for older people in acute hospitals: a mixed-methods study. Health Services and Delivery Research 2013; Volume 1, Issue 11.

Hill SR, Mason H, Poole M, Vale L, Robinson AL. What is important at the end of life for people with dementia? The views of people with dementia and their carers. International Journal of Geriatric Psychiatry 2017; 32: 1037-1045.

Jackson TA, Gladman JRF, Harwood RH, et al. Challenges and opportunities in understanding dementia and delirium in the acute hospital. PLoS Med 2017; 14(3): e1002247.

Murray SA, Kendall M, Boyd K, Sheikh A. Illness trajectories and palliative care. BMJ 2005; 330: 1007-1011.

Nicholson C, Meyer J, Flatley M, Holman C, Lowton K. Living on the margin: understanding the experience of living and dying with frailty in old age. Social Science and Medicine 2012; 75: 1426-32.

Office for National Statistics. Life tables for England 2014-16. Available at https://www.ons.gov.uk/ (accessed 24 ${ }^{\text {th }}$ August 2018).

Partridge JS, Harari D, Martin FC, et al. Randomized clinical trial of comprehensive geriatric assessment and optimization in vascular surgery. British Journal of Surgery 2017; 104:679687.

Pollock K. Is home always the best and preferred place of death? BMJ 2015;351:h4855 
Porock D, Clissett P, Harwood RH, Gladman JRF. Disruption, control and coping: responses of and to the person with dementia in hospital. Ageing and Society 2014, 1- 27

Reynish EL, Hapca SM, De Souza N, Cvoro V, Donnan PT, Guthrie B. Epidemiology and outcomes of people with dementia, delirium, and unspecified cognitive impairment in the general hospital: prospective cohort study of 10,014 admissions. BMC Medicine 2017; $15: 140$

Reljic T, Kumar A, Klocksieben FA, et al. Treatment targeted at underlying disease versus palliative care in terminally ill patients: a systematic review. BMJ Open 2017;7:e014661.

Roche JJW, Wenn RT, Sahota O, Moran CG. Effect of comorbidities and postoperative complications on mortality after hip fracture in elderly people: prospective observational cohort study. BMJ 2005; 331:1374

Rolfson DB, Majumdar SR, Tsuyuki RT, Tahir A, Rockwood K. Validity and reliability of the Edmonton Frail Scale. Age and Ageing 2006; 35: 526-529.

Siddiqi $\mathrm{N}$, House AO, Holmes JD. Occurrence and outcome of delirium in medical in-patients: a systematic literature review. Age and Ageing 2006; 35: 350-64.

Temel JS, Greer JA, Muzikansky A, et al. Early palliative care for patients with metastatic non-small-cell lung cancer, N Engl J Med 2010; 363: 733-742.

Whittamore K, Goldberg S, Gladman JRF, Jones RG, Harwood RH. The diagnosis, prevalence and outcome of delirium in a cohort of older people with mental health problems on general hospital wards. International Journal of Geriatric Psychiatry 2014; 29:32-40. 
Table 1: Dimension of Comprehensive Geriatric Assessment (CGA)

\begin{tabular}{|l|l|}
\hline Dimension & Comments \\
\hline Medical & $\begin{array}{l}\text { Acute and chronic diagnoses, multi-morbidity, clinical therapeutics and } \\
\text { polypharmacy }\end{array}$ \\
\hline Functional & Mobility, continence, activities of daily living, behaviour \\
\hline Mental health & $\begin{array}{l}\text { Psychiatric diagnoses, including delirium, dementia, and depression, and } \\
\text { psychological response to illness }\end{array}$ \\
\hline Social & Relationships, resources, and support networks \\
\hline Environmental & Type of accommodation, including care homes, physical environment \\
\hline
\end{tabular}

Table 2: Goals of healthcare

\begin{tabular}{|l|}
\hline Goal \\
\hline Prevention (of disease or complications of disease or therapy) \\
\hline Cure (of disease or complications of disease or therapy) \\
\hline Delaying death \\
\hline Relief of unpleasant symptoms \\
\hline Maximising physical and social function \\
\hline Provision of information (diagnosis, prognosis, treatment options) \\
\hline Support for families and other carers \\
\hline
\end{tabular}

Table 3: Models of medical care

\begin{tabular}{|l|l|}
\hline Model & Approach \\
\hline Prevention & $\begin{array}{l}\text { Intervention, with drugs or to change habits or lifestyle, to reduce the } \\
\text { occurrence or recurrence of a disease, especially in those identifies as at } \\
\text { increased risk }\end{array}$ \\
\hline Acute medical & $\begin{array}{l}\text { Explanation of problems by diagnoses, and use of specific drug or surgical } \\
\text { therapy to cure or mitigate the effects of the underlying disease }\end{array}$ \\
\hline Rehabilitation & $\begin{array}{l}\text { Restoration or maximising of functional ability. Reablement (restoration of } \\
\text { abilities), resettlement (adaptation of the physical and social environment to } \\
\text { compensate for problems) and readjustment (resetting of ambitions and goals, } \\
\text { and coming to terms with changed ability), through a multi-disciplinary } \\
\text { problem-solving approach }\end{array}$ \\
\hline $\begin{array}{l}\text { Person-centred } \\
\text { dementia care }\end{array}$ & $\begin{array}{l}\text { Enhancing the wellbeing and personhood of someone living with dementia, } \\
\text { through supporting identity, comfort, inclusion, attachment, and occupation }\end{array}$ \\
\hline Palliative & $\begin{array}{l}\text { An approach that improves the quality of life of patients and their families } \\
\text { facing the problems associated with life-threatening illness, through the } \\
\text { prevention and relief of suffering by means of early identification and } \\
\text { impeccable assessment and treatment of pain and other problems, physical, } \\
\text { psychosocial and spiritual }\end{array}$ \\
\hline Social & $\begin{array}{l}\text { Adaptation of the environment, and provision of human help, aids or } \\
\text { appliances, to compensate for the effects of for disability, infirmity or old age }\end{array}$ \\
\hline
\end{tabular}

